\title{
Incertidumbre ante la enfermedad. Aplicación de la teoría para el cuidado enfermero
}

\author{
Fernando Trejo Martínez*
}

\begin{abstract}
RESUMEN
La teoría de rango medio, propuesta por la Dra. Mishel, evalúa cuáles son las reacciones que las personas tienen ante la enfermedad, ya sea la propia o la de un ser querido, para lo cual conceptualiza el nivel de incertidumbre ante la enfermedad; por ejemplo, la incapacidad de la persona de determinar el significado de los hechos relacionados con la enfermedad, siendo incapaz de dar valores definidos a los objetos o hechos y por lo tanto no puede predecir los resultados; este fenómeno es motivado por la falta de información y/o conocimiento. Si el evento es evaluado de forma efectiva, entonces ocurrirá la adaptación. La dificultad de la adaptación dependerá de la habilidad de las estrategias recopiladas para manipular la incertidumbre en la dirección deseada, ya sea para mantenerla, si se valora como oportunidad, o para reproducirla, si se valora como un peligro. Así mismo, la teoría puede ser utilizada en una amplia gama de posibilidades, ya que tanto la persona enferma, como sus familiares, se enfrentan a situaciones estresantes generadoras de incertidumbre. Es por esto que el profesional de enfermería, al conocer la teoría, puede guiar u orientar a la persona para que enfoque su evaluación como una oportunidad, y dirija sus estrategias a lograr la adaptación.
\end{abstract}

Palabras clave: Cuidado enfermero, incertidumbre ante la enfermedad, Merle Mishel.

\section{Uncertainty in illness. Application nurse care theory}

\begin{abstract}
The middle-range theory proposed by Dr. Mishel, which are evaluated reactions taking people to the disease, either their own or a loved one, for which conceptualizes the level of uncertainty in illness as: the inability of the person to determine the significance of the facts related to the disease, being unable to give definite values to objects or events and therefore can not predict the outcome, a phenomenon caused by a lack of information and / or knowledge. So that if the event is effectively evaluated, then the adjustment will occur. The difficulty of adaptation depends on the ability of strategies compiled to handle uncertainty in the desired direction, either to keep it, if we evaluate it as an opportunity, or to play, if we evaluate as a danger. Also the theory can be used in a wide range of possibilities, since both the sick person, and their families face stressful situations that generate uncertainty, which is why the nurse, knowing the theory can guide the person to focus their evaluation as an opportunity, and direct their strategies to achieve adaptation.
\end{abstract}

Key words: Care nurse, uncertainty in illness, Merle Mishel.

*Maestría en Enfermería en Educación.

Correspondencia: Fernando Trejo Martínez

Insurgentes Sur Núm. 3877. Col. La Fama, 14269, México, D.F. Delegación Tlalpan, E-mail: fertre001@yahoo.com

Este artículo puede ser consultado en versión completa en http://www.medigraphic.com/enfermerianeurologica 


\section{LA TEORÍA DE LA INCERTIDUMBRE: MERLE MISHEL}

$\mathrm{L}$ a teoría de la incertidumbre explica cómo las personas procesan cognitivamente los estímulos relacionados con la enfermedad ${ }^{1}$ y construyen un significado en estos eventos. Antes de empezar a describir la teoría, es necesario definir qué es la incertidumbre. ${ }^{2}$

El Diccionario Enciclopédico Norma define la palabra "incertidumbre" como: tener duda sobre algo; falta de seguridad. ${ }^{3}$ El Diccionario de la Real Academia Española la define como: la falta de certidumbre. ${ }^{4}$ De acuerdo con Abbagnano, la certidumbre tiene dos significados: por un lado, seguridad subjetiva de la verdad de un conocimiento $\mathrm{y}$, por el otro, es la garantía que un conocimiento ofrece sobre su verdad. ${ }^{5}$

Una síntesis de las tres definiciones de la palabra "incertidumbre" puede ser: tener duda sobre la garantía subjetiva que un conocimiento ofrece de su verdad.

Por su parte, Mishel relaciona la incertidumbre con la teoría enfermera y nos ofrece la siguiente definición: la incapacidad para determinar el significado de los eventos relacionados con la enfermedad. ${ }^{1}$ Esto se relaciona con lo mencionado por Calvo en el texto La ciencia y el imaginario social, en donde comenta que "Tanto en la práctica científica como en la vida cotidiana, los conocimientos, las creencias y las teorías que ya sustentamos juegan un papel fundamental en la determinación de lo que percibimos. Es necesario el saber previo para poder interpretar los hechos que se van presentando. El reconocimiento o la interpretación preliminar de algo suponen su inserción en un marco conceptual preexistente, en un cuerpo de información relevante; si falta ese marco, el observador se encuentra en un callejón sin salida." ${ }^{\prime}$

Esta reflexión hace referencia a los dos primeros elementos de la teoría de Mishel debido a que la persona tiene un marco conceptual sobre la enfermedad, o sea, un significado e imaginario que le da pauta para percibir de cierta manera la enfermedad $y$, a su vez, elementos para poder evaluar dicho proceso; esto origina que pueda emitir dos respuestas (según Mishel): la oportunidad y la amenaza. Como se puede observar, lo mencionado por Calvo sintetiza conceptualmente la teoría de la incertidumbre. A continuación presentamos la teoría propuesta por la Dra. Mishel.

El marco estructural de la teoría se compone de tres elementos principales:

1. Antecedentes de incertidumbre

2. El proceso de valoración

3. Enfrentamiento de la enfermedad
1. Antecedentes de incertidumbre: Se refiere al marco de estímulos; a la forma, composición y estructura de los estímulos que la persona percibe. El marco de estímulos tiene, a su vez, tres componentes:

- El patrón de síntomas

- La familiaridad con el evento

- Congruencia con el evento

Estos tres elementos proveen los estímulos que son estructurados por la persona en un esquema que crea menos incertidumbre.

El patrón de síntomas: Se refiere al grado en el cual los síntomas se presentan con suficiente consistencia para percibir que tienen un patrón o configuración. Basados en este patrón, el significado de los síntomas puede ser determinado.

La familiaridad con el evento: Es el grado en el cual la situación es habitual, receptiva o contiene claves reconocidas. Cuando los eventos se reconocen como familiares, se pueden asociar con eventos de la memoria y su significado puede ser determinado.

La congruencia con el evento: Se refiere a la consistencia entre los eventos relacionados con la enfermedad, e implica confiabilidad y estabilidad de los eventos, facilitando así su interpretación y comprensión.

Estos componentes del marco de estímulos están inversamente relacionados con la incertidumbre; ellos reducen la incertidumbre.

A su vez, estos elementos del marco de estímulos están influenciados por dos variables.

A. La capacidad cognitiva

B. Proveedores de estructura

A. Capacidad cognitiva: Son las habilidades de procesamiento de la información de la persona; sólo una capacidad limitada de información puede ser procesada en un determinado momento; la sobrecarga de información ocurre cuando esta capacidad es excedida y la limitación de la capacidad cognitiva reducirá la habilidad para percibir el patrón de síntomas, la familiaridad con el evento y la congruencia con el mismo, lo cual favorece la aparición de la incertidumbre.

B. Proveedores de estructura: Son los recursos disponibles para ayudar a la persona en la interpretación del marco de estímulos, éstos reducen el estado de incertidumbre, tanto directa como indirectamente. La reducción de la incertidumbre ocurre indirectamente cuando los proveedores de estructura ayudan a la persona a determinar el patrón de los síntomas, 
la familiaridad con los eventos y la congruencia de experiencias. Los proveedores de estructura son autoridades confiables por su nivel educativo y apoyo social.

Los estímulos son procesados por el personal para construir un esquema cognitivo para los eventos de enfermedad; en la experiencia de la enfermedad la incertidumbre tiene cuatro formas:

1. Ambigüiedad, relacionada con el estado de enfermedad.

2. Complejidad, con relación al tratamiento y sistema de cuidado.

3. Falta de información, acerca del diagnóstico y la seriedad de la enfermedad.

4. Falta de predicción, sobre el curso de la enfermedad y el pronóstico.

2. El proceso de valoración: Cuando existe la incertidumbre, las tareas preceptuales de reconocimiento y clasificación no se han complementado y la formación del esquema cognitivo está impedida para el reconocimiento y la clasificación de los eventos relacionados con la enfermedad que ocurrirán; los estímulos deben ser específicos, familiares, consistentes, completos, claros y limitados en número. Además, deben corresponder con un marco de referencia existente, congruentes con las expectativas de la persona en un contexto físico y temporal; cuando los estímulos no tienen estas características, ocurren errores en el procesamiento cognitivo y la incertidumbre es el estado cognitivo predominante. El estado de incertidumbre debe ser el resultado de la naturaleza de los estímulos; cuando un evento se percibe como incierto, opera una de las siguientes tres situaciones:

a) El evento no es reconocido.

b) El evento es reconocido, pero no clasificado.

c) El evento es reconocido, pero clasificado incorrectamente.

La experiencia de la incertidumbre es neutral, no es una experiencia deseada ni evitada hasta que no es valorada; la valoración de la incertidumbre comprende dos procesos principales:

- La inferencia: Se construye a partir de las disposiciones de la personalidad y de las creencias generales acerca de uno mismo y de la relación que tiene uno con el ambiente. Entre tales recursos destacan: los aprendidos, sentido de destreza o dominio y capa- cidad de controlar. Estas disposiciones se refieren a las creencias que ellos tienen, las destrezas y el comportamiento para enfrentar en forma efectiva los eventos importantes de la vida, y se hacen efectivas al momento de valorar la incertidumbre; sin embargo, para que sean operativos los recursos aprendidos y la sensación de dominio, los eventos deben estar representados objetivamente y corresponder a experiencias pasadas.

Tratándose de alguna enfermedad, los eventos inciertos carecen de claridad y predicción necesarias para una representación objetiva y no pueden corresponder al aprendizaje pasado. Así, los eventos valorados por estas disposiciones de la personalidad pueden ser vistos como una dificultad para manejarlos. Por cierto, hasta peligrosa.

- La ilusión: Se define como la creencia construida sin incertidumbre y ésta puede ser vista con una luz particular con énfasis en los aspectos positivos.

$\mathrm{El}$ autoengrandecimiento de las ilusiones no puede ser construido sin incertidumbre, una vez que una situación es claramente cierta, es difícil o imposible redefinirla.

La construcción de la ilusión refleja apoyo para la anulación y negación. En estas situaciones, el mantenimiento de la ilusión es valioso para proteger a las personas, en los estados iniciales de la amenaza y cuando deben asimilar información que es difícil de aceptar.

3. Enfrentamiento de la enfermedad: Basado en la valoración; la incertidumbre puede ser vista como un peligro o una oportunidad:

- Incertidumbre, peligro y enfrentamiento: Cuando la incertidumbre está considerada como un peligro, la posibilidad de un resultado perjudicial está determinada por la inferencia; una valoración peligrosa ocurre cuando la precisión predictiva de la inferencia es desconocida. La incertidumbre puede asociarse con una visión pesimista y una evaluación negativa del futuro, se asocia con altos niveles de ansiedad y depresión, y pueden influir en la pérdida o ausencia de la credibilidad en las autoridades y falta de cuidado durante el diagnóstico y el tratamiento.

- Enfrentamiento con una valoración de peligro: Los métodos de enfrentamiento se dirigen a reducir la incertidumbre, si es posible, y a manejar las emociones generadas por esta evaluación de peligro. Existen dos estrategias de enfrentamiento: 
I. La movilización, que contiene tres estrategias de acción.

- Directa, es la estrategia menos empleada para reducir la incertidumbre.

- Vigilancia, se refiere a estar a pie de cama de los enfermos, ya que el estar con ellos y observarlos disminuye el estrés del familiar derivado de los síntomas presentados por los enfermos. Está relacionada sólo a los familiares de los enfermos, principalmente niños hospitalizados o enfermos crónicos.

- Búsqueda de información, se considera primaria para reducir la incertidumbre. La información se utiliza para formular tablas de predicciones y probabilidades de tiempo con el fin de formar un marco conceptual que permita organizar las experiencias relacionadas con la enfermedad.

Las personas significativas también ayudan a la reducción de la incertidumbre al proveer información de expertos o interpretación de los eventos. También puede reducir la incertidumbre al estar en contacto con otras personas que se han enfrentado a la misma situación; por ejemplo, los grupos de apoyo o asociaciones de enfermos.

II. El manejo del efecto, que contiene tres métodos

- De fe

- De desapego

- Soporte cognitivo

Los estudios de manejo del efecto no han sido estudiados individualmente sino que se han considerado como un todo, $\mathrm{y}$ existe la posibilidad de que estas estrategias se utilicen para manejar la incertidumbre, aunque existen estudios que han demostrado que las personas enfermas crónicas preferían estas estrategias para controlar las emociones negativas. ${ }^{1}$

- Incertidumbre, oportunidad y enfrentamiento: Cuando la incertidumbre es considerada una oportunidad, la posibilidad de resultados positivos ocurre en respuesta a la valoración; esta posibilidad puede resultar de la inferencia o la ilusión, pero surge en mayor medida del proceso de la ilusión. La naturaleza amorfa y vaga de una situación de incertidumbre permite que ésta sea modificada por las personas en una situación positiva. Bajo la perspectiva de la incertidumbre, una nueva estructura de ilusión puede desarrollarse, lo cual presagia resultados positivos. Ver la incertidumbre como una oportunidad requiere preocuparse más por lo positivo que por lo negativo. Una valoración de oportunidad ocurre cuando existe una alta probabilidad de certidumbre negativa, entendiendo ésta como una situación de enfermedad con una trayectoria de descenso.

Ahora bien, en 1990 Mishel reconceptualizó su teoría, en virtud de un análisis de las experiencias de personas cuando la enfermedad se hace crónica, ${ }^{7}$ debido a que "las reacciones de las personas ante la incertidumbre cambiaban a lo largo del tiempo". ${ }^{8}$ Esta aseveración fundamenta la aplicación de la teoría dirigida a personas con alteraciones neurológicas, y cuidadores de los mismos; además le proporciona al profesional de enfermería una visión a futuro de que las personas no están en un dimensión estática: la incertidumbre se verá modificada de acuerdo a cómo evolucione la enfermedad y puede que los significados de la misma estén en constante reevaluación. A razón de esto es que se debe realizar un plan de cuidados previendo estas circunstancias, y realizando un acompañamiento, identificando cuando la evaluación se está enfocando hacia un peligro en lugar de oportunidad, además de fortalecer con acciones encaminadas a lograr siempre la adaptación de cualquier adversidad o evento que genere una nueva incertidumbre al núcleo familiar del enfermo.

La teoría de la incertidumbre ante la enfermedad brinda al profesional un sinfín de posibilidades de acción y ofrece la libertad de generar una gama de intervenciones enfocadas a la disminución del estrés provocado por la enfermedad, prestando atención a la multiplicidad de dimensiones que un individuo posee; esto suscita que las intervenciones tengan un carácter humanista, lo cual es parte de la esencia de nuestra profesión.

\section{CONCLUSIONES}

Las teorías de enfermería son una herramienta que brinda sustento teórico al profesional de enfermería para desarrollar sus intervenciones y también son una guía en el quehacer cotidiano para desempeñarse con conocimiento, ética y humanismo en el cuidado de las personas. Estas teorías generan, sobre todo, que el profesional de enfermería entienda que interactúa con un ser humano que es indivisible y que posee una gama de dimensiones que necesitan ser abordadas mediante un cuidado holístico. La incertidumbre provocada por la enfermedad puede hacer que las personas evalúen inadecuadamente una situación estresante; la aplicación de la teoría de Mishel conducirá al profesional en la identificación de los primeros datos de incertidumbre para realizar un plan de cuidados enfocados a eliminar esta situación y, posteriormente, lograr que la persona o familiar afronten y se adapten a la enfermedad. 


\section{BIBLIOGRAFÍA}

1. Mishel M. Uncertainity in illness. J Nurs Scholarsh 1988; 20 (4): 225-231.

2. Torres A, Sanhueza O. Modelo estructural de enfermería de calidad de vida e incertidumbre ante la enfermedad. Ciencia y Enfermería 2006; XII (1): 9-17.

3. Diccionario Enciclopédico Ilustrado Norma. Editorial Norma S.A. Colombia 2001: 807.
4. Diccionario de la Real Academia de la Lengua Española, consultado en http://buscon.rae.es/draeI/

5. Abbagnano N. Diccionario de Filosofía. 4ta ed. México: Fondo de Cultura Económica; 2008: 164-165.

6. Díaz E. La ciencia y el imaginario social. Argentina: Biblos; 1996.

7. Aimar A. El cuidado de la incertidumbre en la vida cotidiana de las personas. Index Enfer 2009; 18. Consultado en: http://dx.doi.org/10.4321/ S1132-12962009000200009 el 24 de marzo 2012.

8. Marriner-Tomey A, Alligood MR. Modelos y teorías en enfermería. 5ta ed. Madrid: Mosby; 2003. 